\title{
Spin glasses without time-reversal symmetry and the absence of a genuine structural glass transition
}

\author{
Barbara Drossel \\ School of Physics and Astronomy, Raymond and Beverley Sackler Faculty of Exact Sciences, Tel Aviv University, Tel Aviv \\ 69978, Israel \\ Hemant Bokil* \\ Abdus Salam ICTP, Strada Costiera 11, 34100 Trieste, Italy \\ M. A. Moore \\ Department of Physics, University of Manchester, Manchester M13 9PL, U.K.
}

(October 28, 2018)

\begin{abstract}
We study the three-spin model and the Ising spin glass in a field using Migdal-Kadanoff approximation. The flows of the couplings and fields indicate no phase transition, but they show even for the three-spin model a slow crossover to the asymptotic high-temperature behaviour for strong values of the couplings. We also evaluated a quantity that is a measure of the degree of non-self-averaging, and we found that it can become large for certain ranges of the parameters and the system sizes. For the spin glass in a field the maximum of non-self-averaging follows for given system size a line that resembles the de Almeida-Thouless line. We conclude that non-self-averaging found in MonteCarlo simulations cannot be taken as evidence for the existence of a low-temperature phase with replica-symmetry breaking. Models similar to the three-spin model have been extensively discussed in order to provide a description of structural glasses. Their theory at mean-field level resembles the mode-coupling theory of real glasses. At that level the one-step replica symmetry approach breaking predicts two transitions, the first transition being dynamical and the second thermodynamical. Our results suggest that in real finite dimensional glasses there will be no genuine transitions at all, but that some features of mean-field theory could still provide some useful insights.
\end{abstract}

\section{INTRODUCTION}

Despite over two decades of work, the controversy concerning the nature of the ordered phase of short-range Ising spin glasses continues. Monte-Carlo simulations of three- and four-dimensional systems appear to be providing evidence for replica symmetry breaking (RSB) in these systems (for recent reviews see [1,2]). However, recent developments have cast doubt on this interpretation of the Monte Carlo data. In a series of papers on the Ising spin glass within the Migdal-Kadanoff approximation (MKA), we showed that the equilibrium Monte Carlo data in three and four dimensions that had been interpreted in the past as giving evidence for RSB can actually be interpreted quite easily within the droplet picture, with apparent RSB effects being attributed to a crossover between critical behaviour and the asymptotic droplet-like behaviour for small system sizes [3 [7]. We also showed that system sizes well beyond the reach of current simulations would probably be required in order to unambiguously see droplet-like behaviour. Very recently, a third view on the nature of the low-temperature phase if spin glasses has emerged. In this picture, there exist droplet excitations in short scales, but on large scales there are system-wide excitations that cost only a finite energy in the thermodynamic limit, and that have a surface whose fractal dimension is less than the space dimension 8 12]. It remains to be seen whether these excitations survive at larger system sizes. Within MKA, fractal excitations are not possible, and the signatures of these excitations found in Monte-Carlo simulations are therefore not present in MKA.

There is a close connection between the question of the nature of the spin glass phase and that of the existence of a phase transition in a spin glass in an external field. Mean-field theory predicts a phase transition to a spin glass phase with RSB along the so-called de AlmeidaThouless line. The droplet picture predicts no transition. The reason is that in the presence of a field time reversal symmetry is broken, and there is no symmetry left that could possibly be broken by a phase transition, except for replica symmetry. Monte-Carlo simulation of the spin glass in a field 13 15] show some evidence of a phase transition, and in particular of non-self-averaging, however, the situation is complicated by the presence of

${ }^{*}$ Present Address: Program in Neuroscience, University of Maryland, Baltimore MD 21201, U.S.A. 
large finite-size effects due to crossover phenomena. For this reason, Parisi et al 16 studied a different system that also has broken time-reversal symmetry, but is expected not to have strong crossover effects. This system is the three-spin model, where the two-spin products in the Ising spin glass without field are replaced by three-spin products. The numerical evidence for a phase transition in the four-dimensional system seems good, if a quantity that measures the degree of non-self-averaging is studied.

It is the purpose of this paper to study the two mentioned systems without time-reversal symmetry in MKA, in order to check whether a similar degree of non-selfaveraging could be produced by finite-size effects. The flow of the coupling constants shows that for both systems there exists only one attractive fixed point which corresponds to a paramagnet in a random field, and that there is consequently no phase transition within MKA. Nevertheless, in both systems the coupling constants increase initially for sufficiently low temperatures, indicating that for small system sizes there might be the appearance of a phase transition. Then, we looked at the nonself-averaging parameter in both models within MKA, for various system sizes and parameter values. We found a behaviour similar to the one reported for the MonteCarlo simulations, and apparent RSB for system sizes similar to theirs. Furthermore, for the spin glass in a field, the maximum of the non-self-averaging parameter as function of the field (for fixed system size) marks a line that can be interpreted as a remnant of the de AlmeidaThouless line.

Some insights into what might be expected in the finite dimensional three-spin model can be obtained from the mean-field solution of the p-spin model (for a review of which see [17). It has an analytical solution in the limit where $p$ goes to infinity which can be obtained by a one-step replica symmetry breaking scheme. For our purposes the solution is best understood in terms of metastable states which can be identified with the solutions of Thouless-Anderson-Palmer (TAP)-like equations [18. The partition function is obtained from the integral

$$
Z=\int_{f_{\min }}^{f_{\max }} d f \exp (N \sigma(f)-N f \beta),
$$

where $\beta=1 / k_{B} T$ and $N$ is the number of spins in the system. $f$ is the free energy density of a TAP state. Solutions of the TAP equations exist for $f_{\min }<f<f_{\max }$, and the number of solutions at free energy density $f$ is exponentially large and given by $\exp (N \sigma(f))$, with $\sigma(f)$ vanishing at both $f_{\min }$ and $f_{\max }$. For temperatures in the interval $0<T<T_{S}$ the integral is dominated as $N \rightarrow \infty$ by the lower limit of the integral, i.e. states whose free energy $N f$ differs from the state of lowest energy by only a finite amount. For temperatures in the interval $T_{S}<T<T_{D}$ the integral can again be done by steepest descents and is dominated by some value of $f$ lying in the interval $f_{\min }<f<f_{\max }$. As the temperature approaches $T_{D}$ this value tends to $f_{\max }$. In the tem- perature interval $T_{S}<T<T_{D}$ an exponentially large number of states contribute to the thermodynamics in contrast to the situation below $T_{S}$ where only a finite number contribute. Above $T_{D}$, only the trivial paramagnetic state contributes. There are thus two phase transitions at mean-field level. The lower temperature transition at $T_{S}$ is accompanied by singularities in the free energy but at the higher temperature transition $T_{D}$ the free energy is smooth and the presence of a transition is best inferred from singularities in the dynamics.

Now for a finite dimensional system metastable states with $f>f_{\min }$ are unstable. (Imagine in such a state converting a block of spins of linear dimension $L$ to have the orientations that they would have in the lowest state; the free energy gain will be or order $L^{d}$; the energy cost of creating such a region will be no more than the cost of breaking all the bonds at the surface of the region, $L^{d-1}$. Thus the possibility of nucleating lower free energy states prevents the existence of metastable states in finite dimensional systems). As the transition at the higher temperature $T_{D}$ involves the metastable states only, one deduces that it will not exist in a finite dimensional system. The only transition which could possibly survive to finite dimensions is the one associated with $T_{S}$. Our studies however indicate that it too probably does not occur in finite dimensional systems.

Our study of the three-spin model had another, perhaps physically more significant motivation. The threespin model and its cousins have been extensively studied at mean-field level as models of structural glasses [19]. The higher temperature transition, $T_{D}$, whose signature is purely dynamical (and very similar to that in the mode-coupling approach to real glasses [20]), while the transition at the lower temperature, $T_{S}$, is associated the Kauzmann temperature, $T_{K}$ [21], the temperature at which the configurational entropy of the glass goes to zero. It has been a common belief of many workers for several decades that there is no genuine transition at $T_{K}$. Recently this belief has been strongly reinforced by the novel Monte Carlo simulation of Santen and Krauth [22], who found no evidence of a genuine transition in a simulation in which the particles could be properly equilibriated. Our work strengthens the argument that no genuine transition analogous to $T_{S}$ or $T_{K}$ will exist in finite dimensions. Nevertheless we can see echoes of the mean-field results in our calculations. It is our belief that future work should focus on how the singularities in mean-field results are "rounded-off" in finite dimensions.

This paper is organized as follows: First, we define the models and quantities studied in this paper. Then, we describe the MKA for the two models studied in this paper. We use two different methods in order to make sure that the results do not depend on the particular implementation of the MKA. Next, we study the three-spin model and give our results for the flow of the coupling constants, and for the non-self-averaging parameter. In section $\mathrm{V}$, we discuss the spin glass in an external field. Again, we give results for the flows and the degree of non- 
self-averaging. We also give scaling arguments based on the droplet picture that explain most of our findings. Finally, we summarize and discuss our results.

\section{MODELS AND DEFINITIONS}

The Edwards-Anderson spin-glass Hamiltonian $\mathcal{H}$ in the presence of a uniform external magnetic field $h$ is given by

$$
-\beta \mathcal{H}(\sigma)=\sum_{\langle i, j\rangle} J_{i j} \sigma_{i} \sigma_{j}+h \sum_{i} \sigma_{i}
$$

where the Ising spins $\sigma_{i}$ can take the values \pm 1 , and the nearest-neighbor couplings $J_{i j}$ are independent from each other and Gaussian distributed with a standard deviation $J$. For convenience, the Boltzmann-factor $\beta$ is absorbed into the couplings and fields. Without a field $h$, the model has a low-temperature phase with nonvanishing correlations $\left\langle S_{i} S_{j}\right\rangle$ even for spins that are far apart. According to the droplet picture, this phase is unique (up to a global flip of all spins), and it is destroyed as soon as the field is turned on. The reason is that the field induces regions of a sufficiently large radius to flip if the magnetization of this region opposes the field. The radius $r$ of these regions is obtained from the condition that the gain in magnetic energy, $h r^{d / 2}$ becomes comparable to the loss in coupling energy, $J r^{\theta}$, leading to

$$
r \sim(J / h)^{1 /(d / 2-\theta)} .
$$

Here, $d$ is the dimension of the system, and $\theta$ the scaling dimension of domain walls. Beyond the radius $r$, the long-range correlations of the spin-glass phase are destroyed.

In contrast, the RSB picture predicts the existence of infinitely many different phases of comparable free energy in the absence of a field. With increasing field $h$, the number of phases decreases, and it becomes one at the de Almeida-Thouless line $h_{c}(J)$. At the critical spinglass transition $J_{c}$, the critical field $h_{c}$ vanishes, and it diverges to infinity as the coupling strength $J$ diverges (i.e., as the temperature goes to zero).

The numerical analysis of the spin glass in a field is hampered by strong crossover effects. Crossover effects are expected to be less strong in the three-spin model, because it has no tunable parameter that can restore time reversal symmetry and lead to strong crossover effects when small [15]. In the most easily tractable version of this model, each site is occupied by two Ising spins, $\sigma_{i}^{(1)}$ and $\sigma_{i}^{(2)}$, and the Hamiltonian is given by

$$
\begin{aligned}
-\beta \mathcal{H}(\sigma) & =\sum_{i j}\left(J_{i j}^{(1)} \sigma_{i}^{(1)} \sigma_{i}^{(2)} \sigma_{j}^{(1)}+J_{i j}^{(2)} \sigma_{i}^{(1)} \sigma_{i}^{(2)} \sigma_{j}^{(2)}\right. \\
& \left.+J_{i j}^{(3)} \sigma_{i}^{(1)} \sigma_{j}^{(1)} \sigma_{j}^{(2)}+J_{i j}^{(4)} \sigma_{i}^{(2)} \sigma_{j}^{(1)} \sigma_{j}^{(2)}\right),
\end{aligned}
$$

where $i j$ are nearest-neighbor pairs, and the couplings $J_{i j}^{(n)}$ are chosen independently from a Gaussian distribution with zero mean and width $J$. When the signs of all spins are reversed, the sign of the Hamiltonian changes also, indicating the violation of time-reversal symmetry.

If finite-dimensional systems have no RSB, this model has no phase transition since there is no symmetry that could be broken. On the other hand, if RSB occurs in finite-dimensional spin glasses, the three-spin model could show a phase transition at some critical coupling strength $J_{c}$.

It has proven useful to consider two identical copies (replicas) of the system, with the spins $\left\{\sigma_{i}\right\}$ and $\left\{\tau_{i}\right\}$, and to measure overlaps between them. This gives information about the structure of the low-temperature phase, in particular about the number of pure states. The main quantity studied in this paper is the parameter $A$ which measures the degree of non-self averaging, and is defined by

$$
A=\frac{\left[\left\langle(q-\langle q\rangle)^{2}\right\rangle^{2}\right]}{\left[\left\langle(q-\langle q\rangle)^{2}\right\rangle\right]^{2}}-1,
$$

where $\langle\ldots\rangle$ and $[\ldots]$ denote the thermodynamic and disorder average respectively. The overlap $q$ between the two replicas is given by

$$
q=\frac{1}{N} \sum_{i} \sigma_{i} \tau_{i}
$$

for the Ising spin glass in a field, and by

$$
q=\frac{1}{2 N} \sum_{i}\left(\sigma_{i}^{(1)} \tau_{i}^{(1)}+\sigma_{i}^{(2)} \tau_{i}^{(2)}\right)
$$

for the three-spin model. $N$ is the number of sites in the system.

$A$ is most easily evaluated by introducing a coupling between the two replicas, and by differentiating with respect to it. The Hamiltonian for the coupled system is then

$$
-\beta \mathcal{H}_{\epsilon}(\sigma, \tau)=-\beta \mathcal{H}(\sigma)-\beta \mathcal{H}(\tau)+\epsilon N q .
$$

The mean overlap is given by the expression

$$
\langle q\rangle=\left[\frac{1}{N} \frac{\partial}{\partial \epsilon} \ln Z\right]_{\epsilon=0},
$$

and its variance by

$$
\left\langle(q-\langle q\rangle)^{2}\right\rangle=\left[\frac{1}{N} \frac{\partial^{2}}{\partial \epsilon^{2}} \ln Z\right]_{\epsilon=0} .
$$

$Z$ is the partition function.

In systems with RSB, the probability distribution $P(q)$ of $q$ is broad, and $A$ has a nonzero limit in the limit of infinite system size. On the other hand, in the absence of RSB, each sample has only one, sample-independent 
value of $q$, and $A$ vanishes in the thermodynamic limit. Consequently, an $A$ that increases with increasing system size could be taken as an indicator of RSB. However, we will see in this paper that even systems without RSB can show an increasing $A$ over a wide range of system sizes and parameters.

\section{THE MIGDAL KADANOFF APPROXIMATION}

The Migdal-Kadanoff approximation (MKA) is a realspace renormalization group that gives approximate recursion relations for the various coupling constants. An exact decimation, which consists in taking the trace over all those spins that do not belong to the coarse-grained lattice, generates higher-order couplings between spins of more that two sites, and is therefore untractable. In order to circumvent this problem, the MKA moves the bonds of a hypercubic lattice before each decimation step in such a way that no higher-order couplings can be generated. If the bond-moving shall be symmetric with respect to the different space directions, one ends up with the scheme represented in Figure 11.
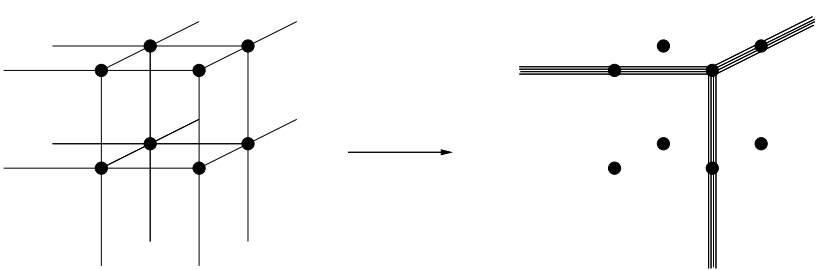

FIG. 1. The Migdal-Kadanoff bond-moving scheme for a cubic lattice.

In a $d$-dimensional lattice, $2^{d-1}$ bonds are superimposed as a consequence of bond-moving. In the absence of field terms (i.e. of terms that couple only spins that sit on the same site $i$, and are therefore not in a clear way associated with bonds), the $2^{d-1}$ coupling constants of each of the $d$ bundles of bonds per coarse-grained unit cell simply add up, and the "naked" spins that are left behind have no couplings. Taking the trace over the $d$ spins that sit on the $d$ main bonds leads to coarse-grained coupling constants between neighboring spins on the coarsegrained lattice. Taking the trace over the naked spins, gives only a constant contribution to the partition function, which can be neglected. This decimation procedure is iterated $n$ times on a lattice of linear size $L=2^{n}$, until a single unit cell is left over. Assuming periodic boundary conditions, one can then take the trace over the final spin.

The flow of the coupling constants in this scheme results from alternating the addition of $2^{d-1}$ bonds with linking two of these new bonds together and taking the trace over the middle spin. Essentially the same flow results when a decimation is done on a hierarchical lattice that is constructed iteratively by replacing each bond by $2^{d}$ bonds, as indicated in Fig. 2. The total number of bonds after $n$ iterations is $2^{d n}$. Spin decimation on such a lattice is done by taking the trace over the spins that are highest on this hierarchy (i.e., that were added last during the construction procedure). At each decimation step, first the trace is taken over the middle spin of two linked bonds, and then $2^{d-1}$ bonds are added together to form a new bond, until the lowest level is reached and the trace over the remaining two spins is calculated [24. Apart from the fact that the order of bond-adding and decimation is reversed, the recursion relations for the coupling constants are obtained by the same procedure as for the bond-moving algorithm.

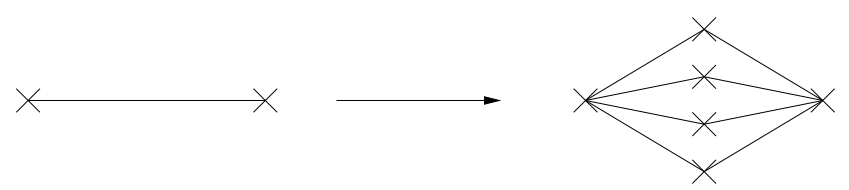

FIG. 2. Construction of a hierarchical lattice.

This equivalence between a bond-moving procedure for a hypercubic lattice and a hierarchical lattice hold no longer when field terms are present. In both of our models, these field terms are present either from the beginning (Ising spin glass in a field), or they are initially absent, but are generated during the decimation procedure (three-spin model). For the bond-moving procedure, one has to decide whether the fields shall also be moved (and to where), or whether they shall remain at their sites, or whether part of them shall be moved. This creates a certain freedom in the renormalization scheme, and the most plausible choice is determined by the requirement that the flows of the fields near the zero-temperature fixed point and at the infinite-temperature fixed point shall be those of the hypercubic lattice. However, if the main results of the MKA shall be generic, they should not depend on the precise implementation of the bond-moving algorithm. Otherwise one might doubt that the MKA reflects the features of the real system. For this reason, we have performed the MKA for a variety of different implementations, and we found our main conclusions concering $A$ to be independent of the implementation. In the following sections, we will give our results for the hierarchical lattice and for a bond-moving scheme that has the correct flow of the field terms.

The treatment of a hierarchical lattice in the presence of field terms is straightforward. In order to understand that the flows of the fields are different on a hierarchical lattice compared to a hypercubic lattice, let us consider a situation where the flows of the couplings go to zero with increasing iteration number, which is the situation that we will encounter below for both models. As long as the couplings are nonzero, each decimation step generates a contribution to the fields at the sites that are left over. The two corner spins, which are left over until the end, receive consequently $2^{n(d-1)}$ field contributions from the first decimation, $2^{(n-1)(d-1)}$ from the second it- 
eration, and so on, until the couplings are virtually zero. For twice the system size, i.e., for a lattice with $n+1$ levels, the mean of the field contribution to the corner spins due to decimations is larger by a factor $2^{(d-1)}$, and so is the variance of the field contribution. Even though the couplings go to zero after a certain number of iterations, the fields keep growing.

In contrast, on a hypercubic lattice, the fields must remain constant as soon as the couplings have become zero. Clearly, this can only be achieved if fields terms are not moved to the sites that will not be traced over. On the other hand, field terms must not be left with the "naked" spins. The reason is that near the zero-temperature fixed point where the couplings are very large, all fields must add up under renormalization. Fields must therefore always stay with spins that are coupled to other spins. For this reason, fields should be moved to those $d$ sites that sit at the middle of the main bonds. Even with this restriction, there remains some freedom in choosing which field should be moved where. In our simulations, we treated field terms as belonging to bonds. The initial fields were evenly distributed between the ends of all bonds, and the fields generated during decimations naturally end up at the ends of bonds. When a bond was moved, we moved all its field terms to that end that was to be traced over next.

For the Ising spin glass in a field, the recursion of four different parameters must be considered when studying the flow diagram and thermodynamic quantities. These are the two-spin coupling, the two fields on the two ends of a bond, and a constant. If one evaluates quantities related to the overlap between two replicas, each site has two spins, leading to 16 parameters. The same number of parameters occurs for the three-spin model, if only one replica is needed, as, e.g., for the flows and the phase diagram. For the evaluation of $A$, we need two replicas, leading 256 couplings. Luckily, the decimation step can treat all 256 parameters with the same formula, which involves a $256 \times 256$ matrix that is calculated once at the beginning of the program.

\section{THE THREE-SPIN MODEL}

This model was studied using Monte-Carlo simulations in four dimensions in [15,23, 16, and evidence for RSB was found. The authors of [23, 16] found in particular that the non-self-averaging parameter $A$ is small for large temperatures, and becomes large for smaller temperatures. Curves for different system size $L=3,4,5,6$ cross nearly at the same temperature, and below this temperature $A$ increases with increasing $L$. Thus, the degree of nonself averaging increases with the system size, just as can be expected for a replica-symmetry breaking transition. Monte-Carlo simulations [15,23, 16] are usually done with couplings $J= \pm 1$. The precise distribution of the couplings should however not affect the universality class.
Analytical results were obtained for the $p$-spin model in mean-field theory, where one-step RSB was found. This means that the ground state has a nonzero probability of being occupied below a critical temperature $T_{S}$ (see Section (i). This mean-field scenario is fundamentally different from the full RSB claimed to be seen in Monte-Carlo simulations of the four-dimensional system. Thus, the argument usually employed for spin glasses that meanfield like behaviour can be found in finite-dimensional short-range systems, fails here.

In the following, we show using MKA that the assumption of the absence of crossover effects in this model is incorrect, and that $A$ might at low temperatures and for small system sizes increase with increasing system size even if the system is self-averaging in the thermodynamic limit. We mainly focus on the case of four dimensions, but report also some results in $\mathrm{d}=2$ and 3 . Let us first discuss the flow of the coupling constants as the system is renormalized. Because each bond is connected to 4 spins, the flow of 16 coupling constants has to be considered. In order to obtain this flow, we iterated the recursion relation on a set of $10^{6}$ bonds. At each iteration, each of the new set of $10^{6}$ bonds was generated by randomly choosing $2^{d}$ bonds from the old set. For a hierarchical lattice, where the generated fields remain at that end of a bond at which they are generated, we first took the trace over the inner spins of each of the $2^{d-1}$ pairs of bonds, and than we added the resulting bonds; for the bond-moving procedure described in the previous section, we first generated two bunches of $2^{d-1}$ bonds each, then moved the fields of all but the "original" bond of each bunch to the inner spin, and took the trace over the inner spin. Figure 3 shows the flow of the width of the three-spin couplings for different initial width in four dimensions, for the two different algorithms.

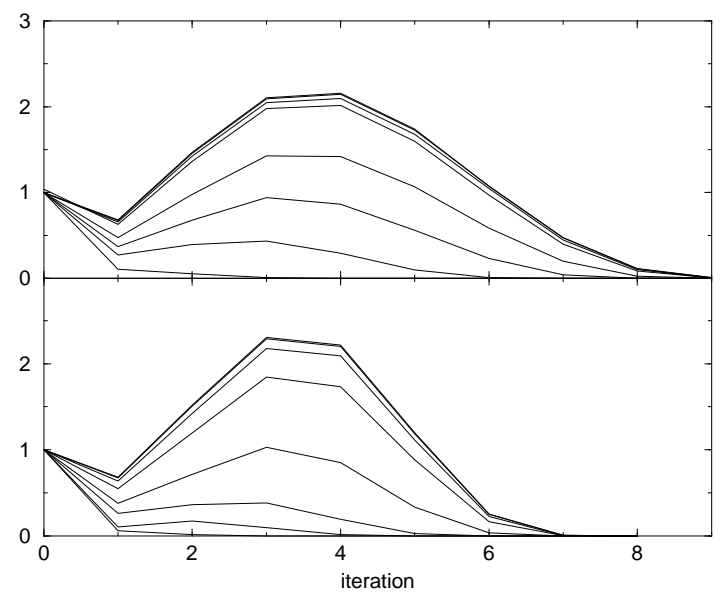

FIG. 3. Flow of the three-spin coupling strength $J$ for bond-moving (top) and the hierarchical lattice (bottom) for $d=4$, divided by the initial coupling strength. The curves correspond to $J=0.065,0.13,0.18,0.26,0.5552,1,2,5$ (bond-moving) and $J=0.13,0.26,0.35,0.52,1,2,5,10$ (hierarchical lattice) from bottom to top. 
One can see that for weak coupling (or, equivalently, high temperature) the coupling strength decreases quickly with increasing system size. However, for stronger coupling or lower temperatures, the coupling strength increases during the first few iterations, and decreases afterwards. The maximum is reached between the 3rd and 4th iteration, or between $L=8$ and $L=16$. For sufficiently strong coupling, the curves reach an asymptotic shape. On the hierarchical lattice, where the fields grow without bounds, the 3 -spin couplings decrease to zero faster than with bond-moving. Furthermore, curves for the hierarchical lattice seem to correspond roughly to those of the bond-moving procedure if the three-spin couplings are divided by a number around 3 . The reason is that the first step during the bond-moving procedure summarizes 8 bonds to one new bond. The width of the three-spin coupling is therefore increased by a factor of $\sqrt{8}$ in four dimensions. In order to compare to the hierarchical lattice or to Monte-Carlo simulations on a hypercubic lattice, one should divide the coupling strength of the bond-moving procedure by $\sqrt{8}$.

If one considered only systems of sizes up to 8 , one would get the illusion of a phase transition with a $(1 / J)_{c}$ around 3 or 4 , a value which is not far from the one given for $T_{c}$ in [16]. (Note that these authors have kept the coupling strength fixed at \pm 1 , and varied the temperature. Their $T$ corresponds therefore to our $1 / J$.)

Figure 4 shows the flow of the widths of the different coupling constants for an initial width of the three-spin coupling $J=2$.

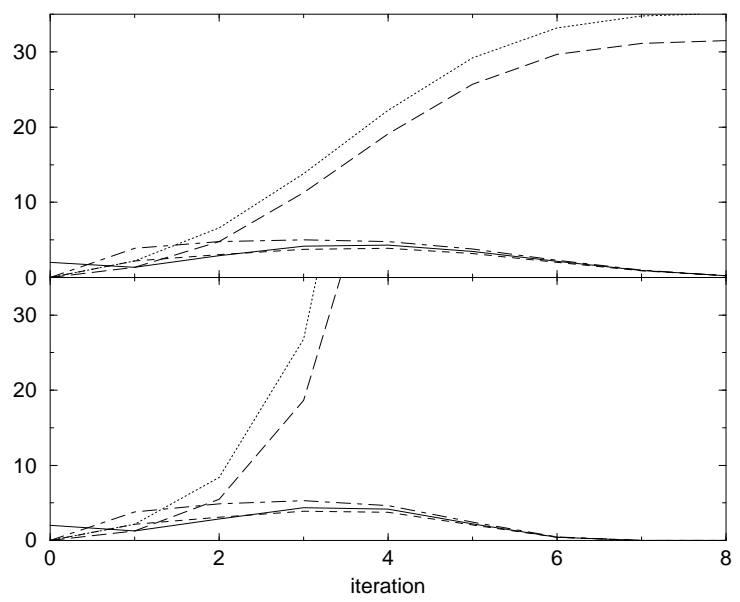

FIG. 4. Flow of the width of the field (long dashed), of the on-site two-spin coupling (dotted), the two-spin coupling across a link (dashed), the three-spin coupling (solid), and the four-spin coupling (dot-dashed) for bondmoving (top) and on the hierarchical lattice (bottom) for $d=4$, for an initial three-spin coupling $J=2$.

One can see that the strengths of the field and of the on-site two-spin coupling (which can also be viewed as a "field") increase rapidly and without limits for the hierarchical lattice, and that they saturate at a finite value in the bond-moving case. The two- three- and four-spin cou- plings increase during the first few iterations, and then decrease again. Thus, our three-spin model corresponds on large scales to a system only with random fields and random couplings between the $\sigma$ and $\tau$ spins on the same site. There are no couplings between spins on different sites on large enough scales, but sites are independent from each other. Only on small scales could one get the impression that the system has long-range correlations. However, these system sizes are exactly the ones studied in 15, 23, 16.

The crossover regime becomes larger with increasing dimension. Figure 5 shows the flow of the three-spin coupling for an initial value $J=10$ in $d=2,3,4$ dimensions. Clearly, the strength of the increase and the range of system sizes over which this increase occurs increases with increasing dimension. One can therefore expect that in even higher dimensions, the apparent phase transition becomes more pronounced.

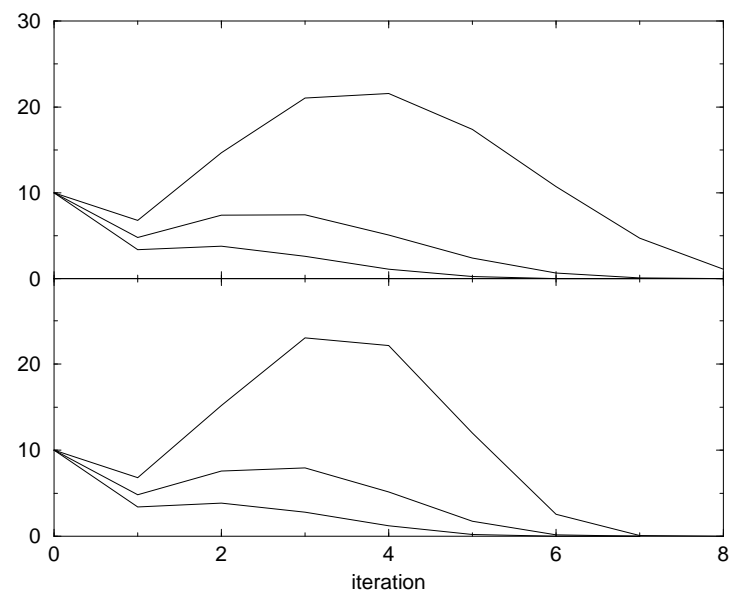

FIG. 5. Flow of the width of the field for an initial value $J=10$ in $d=2,3,4$ dimensions (from bottom to top). The top graph is for bondmoving, the bottom graph for a hierarchical lattice.

Next, let us study the non-self-averaging parameter $A$. As explained in the previous section, $A$ can be evaluated by introducing a coupling between two identical replicas of the system. Since there are now 8 spins associated with each bond, the number of couplings that have to be evaluated in MKA approximation is $2^{8}=256$. Figure 6 shows $A$ as function of the coupling strength for different system sizes up to 16, in 4 dimensions. Larger system sizes could not be studied due to limitations in computer time. One can see that $A$ increases with increasing system size whereever it is appreciably different from zero, and reaches large values. This figure gives the impression that the system shows non self-averaging. Of course, for larger system sizes, $A$ must eventually decrease again since we know that there is self-averaging in the thermodynamic limit. In contrast to the MonteCarlo simulation results [23,16], our curves for $A$ do not intersect at a coupling strength and $A$ value of the order 1. We have performed a similar simulation in $d=2$ di- 
mensions and found that $A$ increases as the system size increases over the range $L=2,4,8,16$. However, for $L=32$ and $L=64, A$ decreases. If we assume that the system size for which $A$ is largest increases with each dimension by a factor 2 , as it does for the flow of the couplings, we can expect that in $d=4$ the system sizes for which a decrease in $A$ can be seen is beyond $L=64$.

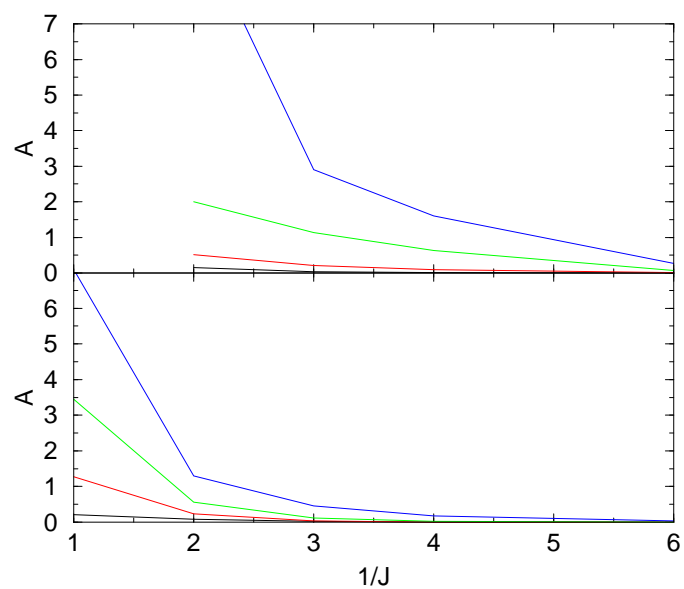

FIG. 6. The non-self-averaging parameter $A$ for $L=2,4,8,16$ (from bottom to top), and $d=4$. The average is taken over 10000 samples for the smallest system size and 100 samples for the largest. The top graph is again for bond-moving, a nd the bottom graph on the hierarchical lattice.

To summarize this section, we have shown that the three-spin model, even in situations where we know that it self-averages in the thermodynamic limit, can show indications of non-self-averaging at those system sizes typically studied in simulations. Evidence for non-selfaveraging found in Monte-Carlo simulations must therefore be taken with caution as it might be misleading.

\section{ISING SPIN GLASS IN A MAGNETIC FIELD}

Monte-Carlo simulations in four dimensions show some indication of RSB [13 15]. Just as for the three-spin model and for the spin glass without external field, these findings may again be due to finite-size effects and to the closeness to the critical temperature. Indeed, an investigation of the ground-state structure of a spin glass in a magnetic field [25] shows no indication of RSB. (See, however, the discussion in 26,27.)

In order to test for finite-size effects, we studied the spin glass in a field using MKA, and determined the non-self-averaging parameter $A$ as function of the system parameters. We found that the degree of non-selfaveraging can be large for the system sizes typically used in simulations, in particular when the contribution of the field to the free energy is comparable to that of the couplings. While most published Monte-Carlo simulations were done in four dimensions, we chose to study the MKA in three dimensions, in order to be able to go to larger system sizes. Because there are three parameters to be varied (the system size, the field, and the two-spin couplings), many data points had to be collected, and this is done faster in 3 dimensions. Of course, we expect that the results of the MKA are similar in four dimensions, if the exponents for 3 dimensions are replaced with those for 4 dimensions. Just as for the three-spin model, the apparent non-self-averaging should become even stronger in 4 dimensions.

First, let us study the flows of the couplings and fields. The decimation procedure leads to the creation of random fields, while the mean value of the field is not changed. Figure 7 shows the flow of the two-spin coupling $J$ for various initial values, and for a fixed field $h=0.1$. For initial couplings larger than the critical coupling (1.13 for the hierarchical lattice and 0.55 for bondmoving), the coupling strength decreases immediately. However, if the initial coupling strength is sufficiently deep in the lowtemperature phase, it increases first, until the random field has become strong enough to have a reducing effect on the coupling strength. Ultimately, the flow goes to a fixed point where the coupling strength is zero. On the hierarchical lattice, the width of the field keeps growing indefinitely, while it saturates in the bond-moving case, as discussed in section III. Clearly, there is no phase transition in the presence of an external field, but there are strong crossover effects if the field is small.

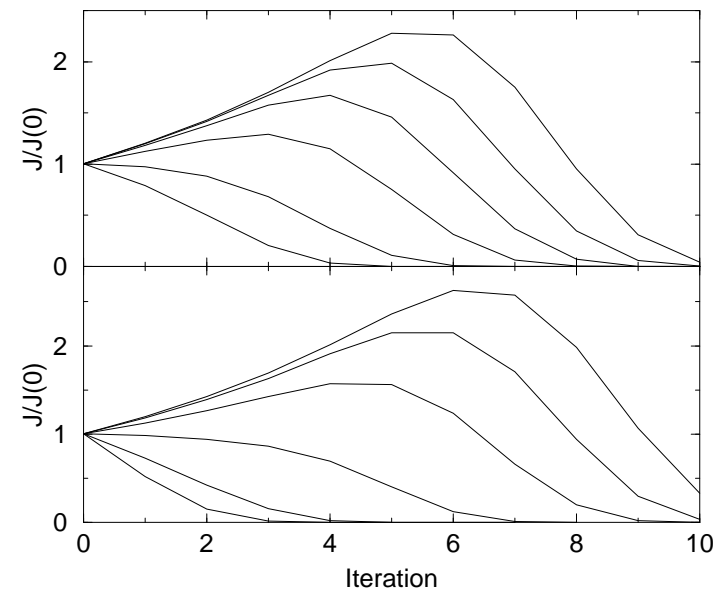

FIG. 7. Flow of the two-spin coupling strength $J$, divided by the initial strenggth, for bond-moving (top) and the hierarchical lattice (bottom) for $d=3$, divided by the initial coupling strength. The curves correspond to $J=0.3,0.5,1,2,4,8$ from bottom to top, for both graphs. The data where obtained from a "cloud" of 50000 bonds.

As mentioned in Section II, the droplet picture predicts that beyond a length scale $r \sim(J / h)^{1 /(d / 2-\theta)}$ the contributions of the field and of the couplings to the free energy become comparable, and we expect that the strength of the couplings decreases beyond this scale. In order to test this prediction, we have plotted in Figure 8 the iteration number for which the two-spin coupling is largest 
versus the logarithm of $J / h$. It should follow a law

$\log _{2} L=\frac{1}{\ln 2((d / 2)-\theta)} \ln (J / h)+C \simeq 1.15 \ln (J / h)+C$,

with a suitable constant $C$. As the figure shows, the data for bond-moving agree nicely with this prediction. for the hierarchical lattice, the slope is larger and has a value around 1.4. This might be due to the fact that the field increases faster on the hierarchical lattice, leading to an earlier reduction in the coupling strength than predicted by the scaling theory.

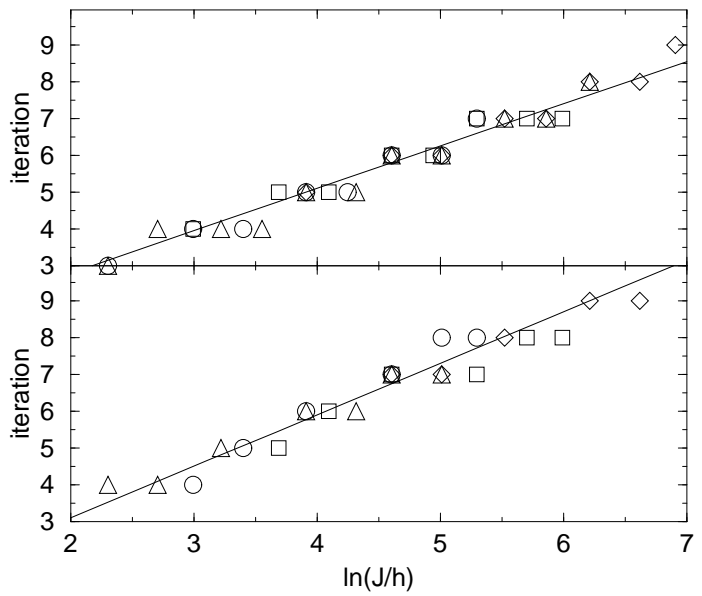

FIG. 8. Iteration number for which the two-spin coupling $J$ reaches its maximum, as function of $\ln (J / h)$, for different values of $h$. The symbols stand for $h=0.2$ (triangle), $h=0.1$ (circle), $h=0.05$ (square), and $h=0.02$ (diamond). The lines have the slope 1.15 and offset $C=0.5$ (bond-moving, top graph), and slope 1.4 and offset $C=0.3$ (hierarchical lattice, bottom graph).

Next, let us discuss the quantity $A$ which is a measure of the degree of non self-averaging. Figure 9 shows our results in the absence of a magnetic field. In the high-temperature phase as well as in the spin-glass phase $A$ descreases with increasing system size and approaches zero, just as one can expect in the absence of RSB. At the critical coupling strength $J_{c}, A$ remains constant with increasing system size, its value being $A \simeq 0.13$ for the hierarchical lattice, and $A \simeq 0.15$ for bondmoving. The constancy of $A$ at the critical point can be explained from the scaling behaviour of the overlap distribution function $P(q)$. Critical scaling implies

$$
[P(q)]=L^{-\beta / \nu}\left[\tilde{P}\left(q L^{\beta / \nu}\right)\right]
$$

and

$$
\left[P(q) P\left(q^{\prime}\right)\right]=L^{-2 \beta / \nu}\left[\tilde{P}\left(q L^{\beta / \nu}\right) \tilde{P}\left(q^{\prime} L^{\beta / \nu}\right)\right],
$$

with $\beta$ being the order parameter critical exponent, and $\nu$ the correlation length exponent. Introducing the vari- able $y=q L^{\beta / \nu}$, we then obtain

$$
A=\frac{\iint y^{2} y^{\prime 2} \tilde{P}(y) \tilde{P}\left(y^{\prime}\right) d y d y^{\prime}}{\left(\int y^{2} \tilde{P}(y) d y\right)^{2}}-1
$$

independently of $L$.

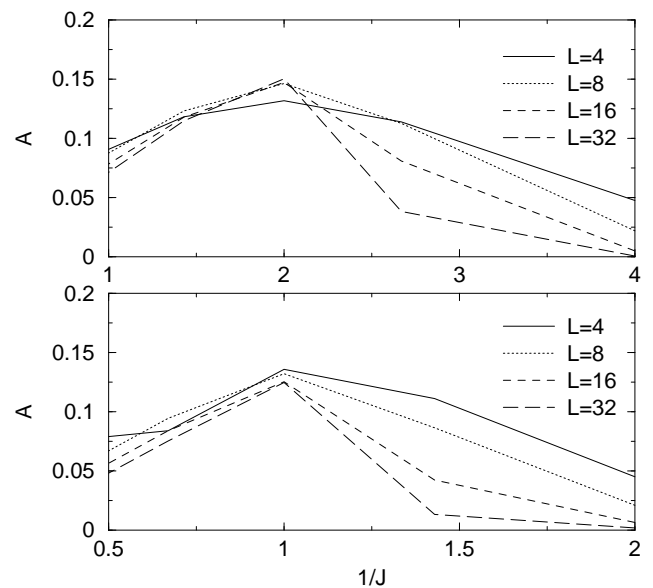

FIG. 9. $A$ as function of $1 / J$ for $h=0$ and $L=4,8,16,32$. The top graph is for bond-moving, the bottom graph on a hierarchical lattice.

For low temperatures $T=1 / J, A$ seems to follow the law $A \sim T L^{-\theta}$ with $\theta \simeq 0.24$. This can be derived by the following argument: At low temperatures, most samples have a value of $\left\langle q^{2}\right\rangle$ close to 1 , and only a fraction $p$ proportional to $k T L^{-\theta}$ of all samples have system-wide excitations and have therefore some other value $\left\langle q^{2}\right\rangle=x<1$. We therefore obtain

$$
\left[\left\langle q^{2}\right\rangle\right] \simeq 1-p+p[x]
$$

and

$$
\left[\left\langle q^{2}\right\rangle^{2}\right] \simeq 1-p+p\left[x^{2}\right]
$$

leading to

$$
A \simeq p\left(1+\left[x^{2}\right]-2[x]\right) \sim k T L^{-\theta} .
$$

In the presence of a magnetic field, we expect $A$ to decrease always to zero for large system sizes, because the system is always in the high-temperature phase without long-range correlations. However, as we will show in the following, $A$ can become nevertheless very large for certain combinations of the system size, the field, and the two-spin coupling strength. One can therefore easily get the impression that the system is not self-averaging, while in reality $A$ increases only over a limited range of system sizes or parameters.

Figure 10 shows our results for $A$ with a magnetic field $h=0.2$. For $J>J_{c}$, the values are larger than without field, and they increase with increasing system size and decreasing temperature. We expect that as the system size increases further, $A$ will reach a maximum and then decrease again. For fields stronger than $h=0.5$, we see 
this reversal in the trend of $A$ already for the system sizes studied in the simulations. For $J<J_{c}$, Fig. 10 shows that the curves for different $L$ intersect each other, such that for high temperatures self-averaging is better for larger system sizes. Thus, the behaviour of $A$ for weak fields seems to be qualitatively similar to that of the three-spin model.

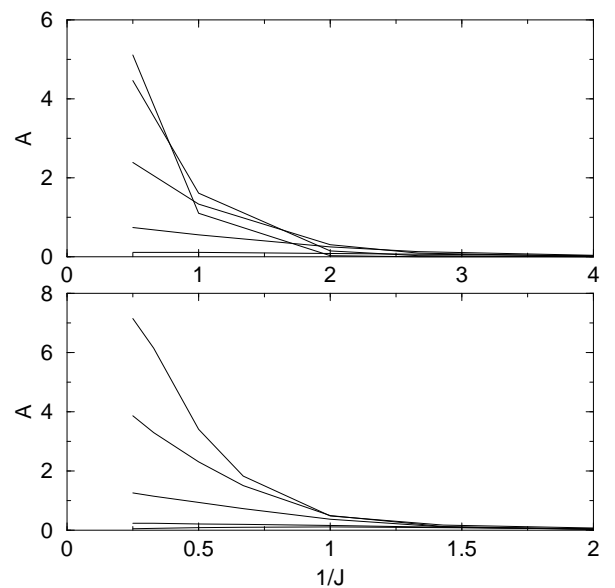

FIG. 10. $A$ as function of $1 / J$ for $h=0.2$ and $L=2,4,8,16,32$ (from bottom to top for the $1 / \mathrm{J}=0.5$ points). The top graph is for bond-moving, the bottom graph on a hierarchical lattice.

For given system size and coupling strength $J>J_{c}$, there exists always a value of $h$ for which $A$ has a maximum. The height of this maximum is higher for larger system sizes and for lower temperatures $1 / J$. Figure 11 shows the field for which $A$ is largest as function of 1/J. The data are in good agreement with a dependence $h_{\max } \propto J$, and $h_{\max } \propto L^{-1.26}$ for bond-moving. This means that $A$ is largest when $h \sim J L^{(d / 2-\theta)}$. For the hierarchical lattice, the fit to the data is best for a dependence $h_{\max } \propto J$, and $h_{\max } \propto L^{-0.93}$. Just as in Figure 8, the effective value of $d / 2-\theta$ appears to be larger on the hierarchical lattice than for bond-moving. We suspect that this is due to the fact that the field grows indefinitely on the hierarchical lattice.

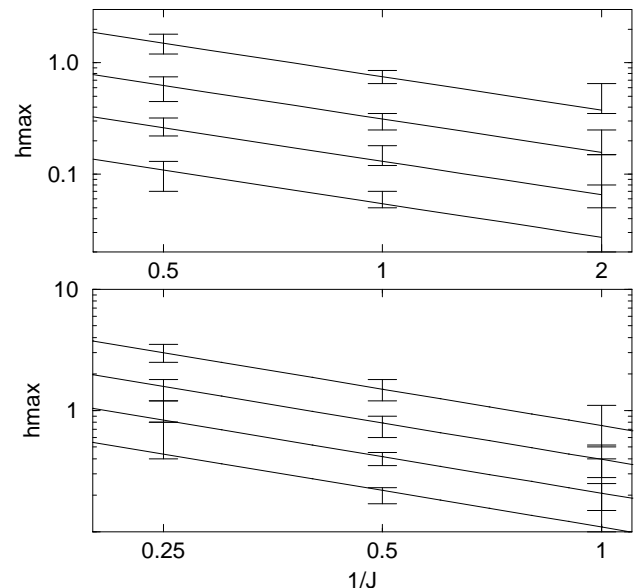

FIG. 11. The field value $h_{\max }$ for which $A$ is largest, for $L=4,8,16,32$ (from top to bottom). The straight lines are power laws $h_{\max } \propto J L^{-1.26}$ (top graph, bond-moving) and $h_{\max } \propto J L^{-0.93}$ (bottom graph, hierarchical lattice).

These results can be understood if one considers the effect of the field on the overlap distribution $P(q)$. Without field, $P(q)$ is a symmetric function, and varies considerably in shape for different samples for the system sizes typically used in simulations. This feature is seen in Monte-Carlo simulations 28] as well as in MKA [3]. A magnetic field changes the shape of $P(q)$ and moves the weight more and more towards positive $q$. In the limit $h \rightarrow \infty$, all spins are aligned with the field, leading to $P(q)=\delta(1)$. Along the boundary line $L \sim$ $(J / h)^{1 /(d / 2-\theta)}$, where the field is not yet strong enough to destroy all features of the low-temperature phase, we can expect that at least some samples still have large droplets than can be flipped without much free energy cost. In Monte-Carlo simulations [14], one finds indeed for certain intermediate parameter values a $[P(q)]$ that has a pronounced peak at some large $q$ value, and a long and thin tail that extends almost all way down to $q=-1$. The authors point out that this feature results from most samples having only the main peak, and other samples having an additional second peak for some other value of $q$. They go on to argue that this is a non self-averaging feature characteristic of RSB, and that it should not be expected if there was no RSB. However, they also admit that their simulations do not show a second peak in $[P(q)]$ at a value $q_{m i n}$, which is expected from MeanField Theory. Although we have not determined $P(q)$ in the presence of a magnetic field within MKA, we can conclude from the behaviour of $A$ that $P(q)$ must have in MKA approximation exactly the same features that we just described for the Monte-Carlo simulations. Indeed, it is easy to show that $A$ becomes large if most samples have a $P(q)$ with one narrow peak at $q_{0}$, and some samples have two peaks. For those samples with one peak, we have a small variance of $q$,

$$
\chi_{s} \equiv\left\langle q^{2}-q_{0}^{2}\right\rangle
$$

which is essentially sample-independent. For those samples with two peaks, we have a large variance $\chi_{l}$ which is different for each sample. If the fraction of samples with two peaks is $p$, we obtain

$$
\begin{aligned}
A & =\frac{(1-p) \chi_{s}^{2}+p\left[\chi_{l}^{2}\right]}{\left((1-p) \chi_{s}+p\left[\chi_{l}\right]\right)^{2}}-1 \\
& \simeq \frac{p\left[\chi_{l}^{2}\right]}{\chi_{s}^{2}+2 p \chi_{s}\left[\chi_{l}\right]+p^{2}\left[\chi_{l}\right]^{2}},
\end{aligned}
$$

where we have only kept the leading terms. As long as $p$ is not much smaller than $\left[\chi_{l}\right] / \chi_{s}, A$ is of the order $1 / p$. Thus, if $\chi_{s}$ is small and $p$ is small but not too small, $A$ is large. The second condition is satisfied if the field is such that a small fraction of samples have a second peak in $P(q)$, the first condition is better satisfied for larger $L$ or 
smaller $T$. This explains why we observe the maximum of $A$ for those $h$ values where the contribution of the field to the free energy is comparable to that of the couplings, and why the maximum of $A$ is larger for larger systems and lower temperatures. Of course, for some even larger value of $L$, we expect $P(q)$ to start having less sample-tosample fluctuations, because the samples should become self-averaging. Then the argument will break down, and $A$ should remain small. However, this range of system sizes seems to be beyond the reach of our simulations.

In conclusion, we have shown that there exists a wide range of parameters over which the degree of non-selfaveraging appears large for system sizes typically used in computer simulations. We expect our results to be valid beyond MKA. As we have argued for systems without a field [3,7], the apparent non-self-averaging must be attributed to the influence of the zero-field critical point. This influence reaches surprisingly far and creates a line in the $h-J$ plane along which non-self-averaging is particularly large, and which is somewhat reminiscent of the de Almeida-Thouless line. We are here in agreement with Huse and Fisher [29] who argued already almost 10 years ago that Monte-Carlo simulation data for a spin glass in a magnetic field are strongly affected by the critical point.

\section{DISCUSSION}

We have shown that for the three-spin model as well as for the spin glass in a magnetic field, a large degree of non-self-averaging found in computer simulations does not represent unequivocal evidence for RSB, but can be caused by finite-size effects.

It seems, however, that a study of the non-selfaveraging parameter $A$ using Monte-Carlo simulations might be able to discriminate between RSB and the droplet picture for three- and four-dimensional spin glasses. As we have shown, $A$ has in zero field a maximum at $T_{c}$, and decreases again with decreasing temperature in MKA. If there was a low-temperature phase with RSB, the low-temperature value for $A$ should probably be larger than the critical value, and $A$ should therefore increase with decreasing temperature. Also, for temperatures below $T_{c}$, we found that $A$ has its maximum not at zero field, but at some finite field value. The degree of self-averaging decreases deep in the supposed low-temperature phase. We expect a similar behaviour from the Monte-Carlo simulations. This would be a hint that non-self-averaging is strongest along the boundary between the field-dominated and coupling-dominated regime, and not in the region where one would expect a low-temperature phase with RSB.

\section{ACKNOWLEDGMENTS}

This work began when HB and BD were at the Department of Physics, University of Manchester, supported by EPSRC Grants GR/K79307 and GR/L38578. BD also acknowledges support from the Minerva foundation, and from the German Science Foundation (DFG, Grant Dr300/2-1), and she acknowledges the hospitality of the ICTP in Trieste during a short visit there. MAM thanks Dr A. Cavagna for useful discussions on the mean-field limit.

[1] E. Marinari, G. Parisi, F. Ricci-Tersenghi, and J.J. RuizLorenzo in (Spin Glasses and Random Fields, ed: A. P. Young), (World Scientific, Singapore, 1997).

[2] E. Marinari, G. Parisi, F. Ricci-Tersenghi, J. RuizLorenzo, F. Zuliani, J. Stat. Phys. 98, 973 (2000).

[3] M. A. Moore, H. Bokil and B.Drossel, Phys. Rev. Lett. 81, 4252 (1998).

[4] H. Bokil, A. J. Bray, B.Drossel, M. A. Moore, Phys. Rev. Lett. 82, 5174 (1999).

[5] H. Bokil, A. J. Bray, B.Drossel, M. A. Moore, Phys. Rev. Lett. 82, 5177 (1999).

[6] Barbara Drossel, Hemant Bokil, M. A. Moore, and A. J. Bray, European Physical Journal B 13, 369-375 (2000).

[7] Hemant Bokil, Barbara Drossel, and M.A. Moore, Phys. Rev. B 62, xxx (2000).

[8] J. Houdayer and O.C. Martin, cond-mat/990878.

[9] J.P. Bouchaud, cond-mat/9910387.

[10] F. Krzakala and O.C. Martin, cond-mat/0002055.

[11] M. Palassini and A.P. Young, cond-mat/0002134.

[12] H.G. Katzgraber, M. Palassini, and A.P. Young, condmat/000713.

[13] E. Marinari, G. Parisi, and F. Zuliani, J. Phys. A: Math. Gen. 31, 1881 (1998).

[14] E. Marinari, C. Naitza, and F. Zuliani, J. Phys. A: Math. Gen. 31, 6355 (1998).

[15] M. Marinari, C. Naitza, F. Zuliani, G. Parisi, M. Picco, F. Ritort, Phys. Rev. Lett. 81, 1698 (1998).

[16] G. Parisi, M. Picco, F. Ritort, Phys. Rev. E 60, 58 (1999).

[17] "Out of Equilibrium Dynamics in Spin Glasses and other Glassy Systems" in "Spin Glasses and Random Fields" (World Scientific 1998, p161, ed A. P. Young), by JP Bouchaud, L. F. Cugliandolo, J. Kurchan and M. Mezard.

[18] T. R. Kirkpatrick and P. G. Wolynes, Phys. Rev. B 36, 8552 (1987).

[19] T. R. Kirkpatrick and D. Thirumalai, Phys. Rev. B 36, 5388 (1987); T. R. Kirkpatrick and P. Wolynes, Phys. Rev A. 35, 3072,(1987).

[20] W. Götze and L. Sjögren, Rep. Prog. Phys. 55, 241 (1992).

[21] W. Kauzmann, Chem. Rev. 43, 219 (1948). 
[22] L. Santen and W. Krauth, Nature, 405, 550 (2000).

[23] M. Marinari, C. Naitza, F. Zuliani, G. Parisi, M. Picco, F. Ritort, Phys. Rev. Lett. 82, 5175 (1999).

[24] B.W. Southern and A.P. Young, J. Phys. C 10, 2179 (1977).

[25] J. Houdayer and O.C. Martin, Phys. Rev. Lett. 82, 4934 (1999).
[26] E. Marinari, G. Parisi, and F. Zuliani, Phys. Rev. Lett. 84, 1056 (2000).

[27] J. Houdayer and O.C. Martin, Phys. Rev. Lett. 84, 1057 (2000).

[28] E. Marinari, G. Parisi, and J.J. Ruiz-Lorenzo, Phys. Rev. B 58, 14852 (1998).

[29] D.A. Huse and D.S. Fisher, J. Phys. I 1, 621 (1991). 